\title{
In vitro and in vivo effect of eco-friendly chemicals on alternaria blight disease (Alternaria brassicae) and yield attributes in Indian mustard (Brassica juncea)
}

\author{
Amarendra Kumar ${ }^{1 *}$, Santosh Kumar ${ }^{1}$, Rakesh Kumar ${ }^{2}$, Gireesh Chand ${ }^{1}$ and S. J. Kolte ${ }^{3}$ \\ ${ }^{1}$ Department of Plant Pathology, Bihar Agricultural University, Sabour, Bhagalpur-821310 (Bihar), INDIA \\ ${ }^{2}$ Department of Soil Science and Agricultural Chemistry, Bihar Agricultural University, Sabour, Bhagalpur-821310 \\ (Bihar), INDIA \\ ${ }^{3}$ Department of Plant Pathology, Govind Ballabh Pant University of Agriculture \& Technology, Pantnagar -263145 \\ (Uttarakhand), INDIA \\ *Corresponding author. E-mail: kumaramar05@gmail.com
}

Received: September 23, 2014; Revised received: February 15, 2015; Accepted: February 16, 2015

\begin{abstract}
The present investigation was done to evaluate the effect of different concentrations of five eco-friendly chemicals in vitro and in vivo, on the management of alternaria blight and yield attributes in Indian mustard (Brassica juncea cv. Varuna). Out of five eco-friendly chemicals, $\mathrm{K}_{2} \mathrm{SO}_{4} 1000$ ppm (64.28\%) followed by $\mathrm{ZnSO}_{4} 1000$ ppm $(63.88 \%)$ showed maximum inhibition of mycelial growth in comparison to check. $0.5 \%$ concentration of $\mathrm{KCl}$ $(57.06 \%)$ followed by $\mathrm{CaSO}_{4}(59.50 \%)$ and $\mathrm{K}_{2} \mathrm{SO}_{4}(62.20 \%)$ showed significantly maximum effect on spore germination in comparison to check (74.60\%). Spore intensity significantly increased by all the treatments except $\mathrm{CaSO}_{4}$ at $0.5 \%$ (40.18\%) followed by $\mathrm{K}_{2} \mathrm{SO}_{4}$ at $0.5 \%(29.86 \%)$ and $\mathrm{ZnSO}_{4} 0.75 \%$ (5.11\% reduction) in comparison to check. The significantly minimum disease index on leaf over check was found by foliar spray of $\mathrm{CaSO}_{4}$ at $0.5 \%(23.58 \%)$ followed by $\mathrm{CaSO}_{4}$ at $1.5 \%(24.00 \%)$ and $\mathrm{Na}_{2} \mathrm{~B}_{4} \mathrm{O}_{7} .10 \mathrm{H}_{2} \mathrm{O}$ at $1.5 \%(24.08 \%) . \mathrm{Na}_{2} \mathrm{~B}_{4} \mathrm{O}_{7} .10 \mathrm{H}_{2} \mathrm{O}$ at $0.75 \%$ showed significantly lowest disease index $(23.91 \%)$ on pod followed by $\mathrm{K}_{2} \mathrm{SO}_{4}$ at $1.5 \%(25.75 \%)$ and $\mathrm{KCl}$ at $1.5 \%(26.00 \%)$ in comparison to check. $\mathrm{CaSO}_{4}$ at $1.0 \%$ showed maximum number of primary branches (7.00), number of secondary branches $(13.00)$ and total yield/ha $(1917.30 \mathrm{~kg} / \mathrm{ha})$ in comparison to check. The results obtained from the present study suggested that $\mathrm{K}_{2} \mathrm{SO}_{4}$ showed maximum in vitro effect on Alternaria brassicae and $\mathrm{CaSO}_{4}$ and $\mathrm{Na}_{2} \mathrm{~B}_{4} \mathrm{O}_{7} .10 \mathrm{H}_{2} \mathrm{O}$ are providing maximum reduction of disease and increase in seed yield/ha that leads to efficient alternaria blight disease management strategies in field condition. These eco-friendly chemicals can protect the crops from alternaria blight diseases and increase the production and productivity of the Indian mustard crop.
\end{abstract}

Keywords: Alternaria blight, Alternaria brassicae (Berk.) Sacc, Eco-friendly chemicals, Mustard

\section{INTRODUCTION}

Rapeseed and mustard (Brassica spp.) are contributing approximately 25 percent of the India's total oilseed production. Among the oilseed crops, India produced $8.2 \mathrm{mt}$ of rapeseed-mustard from $6.7 \mathrm{mha}$ of land during the 2010-11 seasons (GoI, 2011). Among the diseases, alternaria blight disease caused by Alternaria brassicae (Berk.) Sacc has been reported from all the continents of the world and is considered an important constraint in production and productivity of oilseed Brassicas in India, causing up to $47 \%$ yield losses (Kolte et al., 1987; Meena et al., 2012). Average yield losses have been reported in the range of 10-70 per cent (Ram and Chouhan, 1998; Shrestha et al., 2005), depending upon prevailing weather and disease situation (Meena et al., 2004; Chattopadhyay et al., 2005). In addition to the direct losses in yield, the disease adversely affects the seed quality by reducing seed size, seed discolouration and reduction in oil content (Kaushik et al., 1984;
Prasad and Lallu, 2006). Alternaria blight disease can be managed by the use of different fungicides viz. Dithane M 45, Rovral 50 , Ridomil MZ etc. (Verma and Saharan, 1994; Khan et al., 2007; Sultana et al., 2009) that exerted tremendous pressure on environment and human health.

An increasing consciousness about environmental pollution due to pesticides, human health and development of fungicide-resistant strain in plant pathogens has challenged the plant pathologists to search for eco-friendly non-toxic fungicides for substituting the recommended fungicides in recent years (Meena et al., 2013). Mineral nutrition has long been recognized as an important component of disease management practices (Agrios, 2005). A number of trace elements that are not recognized as essential to plants strongly influence the host-pathogen relationships. Macronutrients and micronutrients can be identified with specific biochemical pathways and their effects on disease offer avenues for elucidating mechanisms of resistance in higher plants. In contrast 
to the largely structural, conformational, and osmotic roles of the macronutrients, the micronutrients act as catalysts, cofactors, and inhibitors (Graham, 1983). The eco-friendly approach to overcome biotic stress helped to optimize the economic yields and enable farmers to grow healthy Indian mustard crops (Meena et al., 2011; Meena et al., 2013; Kumar et al., 2014). The present study has been performed to obtain suitable information regarding disease management and precise estimates for yields due to different treatments in Indian mustard. This paper reports in vitro and in vivo effect of eco-friendly chemicals on management of alternaria blight and yield attributes in Indian mustard.

\section{MATERIALS AND METHODS}

Pathogenic fungi: Alternaria brassicae infected leaves exhibiting concentric rings, a typical symptoms of alternaria blight, was collected from the field-grown plants of highly susceptible $B$. juncea $\mathrm{cv}$. Varuna from Crop Research Centre, Govind Ballabh Pant University of Agriculture and Technology, Pantnagar (India). The culture of $A$. brassicae was isolated on potato sucrose agar (PSA) and purified by single spore isolation. The pure culture was incubated at $22 \pm 1{ }^{\circ} \mathrm{C}$, with 12 $\mathrm{hrs}$ light and $12 \mathrm{hrs}$ dark. Pathogenicity of the fungal culture was done by infecting 25 days old plants of highly susceptible $B$. juncea.

In vitro evaluation of eco-friendly chemicals against A. brassicae: The inhibitory effects of four concentrations i.e. 100,500 and $500 \mathrm{ppm}$ of five eco-friendly chemicals viz. $\mathrm{CaSO}_{4}, \mathrm{KCl}, \mathrm{K}_{2} \mathrm{SO}_{4}, \mathrm{ZnSO}_{4} \cdot 7 \mathrm{H}_{2} \mathrm{O}, \mathrm{Na}_{2} \mathrm{~B}_{4} \mathrm{O}_{7} \cdot 10 \mathrm{H}_{2} \mathrm{O}$ and one fungicide, mancozeb as check, were studied. Fifteen-milliliter potato sucrose agar (PSA) medium was poured in each petri plate. Medium without any chemical solution served as control. A 6-mm disc of mycelial growth of $A$. brassicae was placed in the centre of each petri plate and three replications of each concentration were kept. The plates were incubated in an incubator (maintained at $20 \pm 2{ }^{\circ} \mathrm{C}$ with $12 \mathrm{hrs}$ fluorescent light). The colony diameter measured after 7 and 14 days after inoculation (DAI). Per cent inhibition over control was assessed by following Nene and Thapliyal (1993).

$\mathrm{R}=\{(\mathrm{C}-\mathrm{T}) / \mathrm{C}\} \times 100$

Where,

$\mathrm{R}=$ Per cent inhibition

$\mathrm{C}=$ Radial growth of pathogen colony in control

$\mathrm{T}=$ Radial growth of pathogen colony in treatments The effect of five chemicals, each at the concentration of $0.5 \%$ on spore germination of $A$. brassicae were investigated on plain water agar plates at $20^{\circ} \mathrm{C}$ by following the agar plate method (Gattani, 1954). Water agar $(1 \%)$ was prepared and sterilized. For this purpose, double strength solution of different chemicals of desired strength i.e. $0.5 \%$ prepared separately in distilled water. The $10^{4}$ spores $\mathrm{ml}^{-1}$ spore suspension was prepared using two-week-old culture in the Blender. One milliliter of each of the spore suspension and chemicals were mixed and poured on the above mentioned water Agar plates and incubated at $20^{\circ} \mathrm{C}$. Germination of spores was recorded after $8 \mathrm{hrs}$ with the help of a compound microscope. Both germinated and un-germinated spores in the several microscopic fields were counted under the low power. In this way total 100 spores were observed for recording effect on spore germination by different chemicals.

Intensity of sporulation of $A$. brassicae on chemically-treated leaves was studied. Infected leaves from each treatment were separated by $6-\mathrm{mm}$ cork borer and surface sterilized with $0.1 \%$ mercuric chloride and further washed in sterilized distilled water, dried with a sterilized blotting paper and kept for incubation in moist chamber for 72 hrs at room temperature at about $20 \pm 2{ }^{\circ} \mathrm{C}$ with continuous light. Sporulation was determined by suspending the sporulating spots in glass vials containing $1 \mathrm{ml}$ of distilled water and lacto phenol $(9: 1 \mathrm{v} / \mathrm{v})$. The glass vials were then shaken vigorously and spots were scrapped with the help of needle. The spores were counted with the help of haemocytometer.

In vivo evaluation of eco-friendly chemicals against A. brassicae: To study the effect of three different concentrations of five chemicals viz., $\mathrm{CaSO}_{4}, \mathrm{KCl}$, $\mathrm{K}_{2} \mathrm{SO}_{4}, \mathrm{ZnSO}_{4}$, and $\mathrm{Na}_{2} \mathrm{~B}_{4} \mathrm{O}_{7} \cdot 10 \mathrm{H}_{2} \mathrm{O}$, a field experiments were conducted at Govind Ballabh Pant University of Agriculture and Technology, Pantnagar (India) which is situated at $29^{\circ} \mathrm{N}$ latitude and $79.73^{\circ} \mathrm{E}$ longitude and 243.80 meter above sea level. Two sprays of each chemical of the respective concentrations were given during the entire growth period of plants with an atomizer. First spray of respective chemicals was given on all the leaves of 25 days old plants and the second spray was given after onset of disease. This experiment was conducted in a randomized block design (RBD) with three replications. The plot size for each treatment was $3 \times 2 \mathrm{~m}^{2}$ where 5 rows of $3 \mathrm{~m}$ length were maintained by keeping row-to -row distance $40 \mathrm{~cm}$ and plant-to-plant distance $10 \mathrm{~cm}$. Recommended dose of fertilizers of NPK $\left(\mathrm{N}_{100}: \mathrm{P}_{40}\right.$ : $\mathrm{K}_{40}$ ) $\mathrm{kg} \mathrm{ha}^{-1}$ were given in the form of urea, single superphosphate and muriate of potash, respectively.

Data's recorded: Average disease index on leaf due to alternaria blight was taken at 10 days interval at 60, 70, 80 and 90 days after sowing (DAS) and average disease index on pods were recorded at 80 DAS. For recording observations, twenty five leaves and twenty five uniform pods were randomly selected from each replication and rated as per $0-5$ rating scale as $0=$ no symptom, $1=1$ $10 \%, 2=11-25 \%, 3=26-50 \%, 4=51-75 \%$ and $5=>75 \%$ (Verma and Saharan, 1994; Conn et al., 1990) and average disease index was calculated by the following McKinney (1923).

The plant growth and yield components viz. primary branches, secondary branches, number of siliqua/plant, Number of seeds/plant, Seed yield/hectare and oil content (\%) were recorded. Five randomly selected plants per plot were taken for observation. The seed samples were analysed for oil content (\%) with the help of nuclear magnetic 
resonance spectroscope (New Port Analyser) in an oven dried seeds at $60^{\circ} \mathrm{C}$.

Statistical analysis: All data were statistically analyzed using an analysis of variance (ANOVA) to determine the least significant difference $(\mathrm{P}<0.05)$. The data collected as percentages were processed by an angular transformation.

\section{RESULTS AND DISCUSSION}

In vitro evaluation of eco-friendly chemicals against A. brassicae: The data given in table 1 indicated that all the treatments had inhibitory effect on mycelial growth of $A$. brassicae. All the treatments were significantly different over check at both stages of observation i.e. 7 and 14 DAI. After 7 DAI, mancozeb1000 ppm showed maximum inhibitory effect ( $83.33 \%$ ) over check. Among the eco-friendly chemicals, zinc sulphate $1000 \mathrm{ppm}$ and borax 100 ppm concentration gave significantly maximum and minimum inhibition of mycelial growth over check i.e. 77.83 percent and 30.36 percent, respectively. The significantly maximum colony diameter was found in Borax $100 \mathrm{ppm}(5.40 \mathrm{~cm})$ followed by $\mathrm{CaSO}_{4} 100 \mathrm{ppm}$ $(5.30 \mathrm{~cm})$ and $\mathrm{CaSO}_{4} 500 \mathrm{ppm}(4.76 \mathrm{~cm})$ at $14 \mathrm{DAI}$ (Table 1). The significantly maximum inhibition (\%) was recorded in mancozeb1000 and 500 ppm (81.48 and $74.74 \%$ ) followed by $\mathrm{K}_{2} \mathrm{SO}_{4} 1000 \mathrm{ppm}(64.28 \%)$ and $\mathrm{ZnSO}_{4} 1000$ ppm (63.88\%).
All five eco-friendly chemicals were observed for their toxic effect on spore germination of $A$. brassicae (Table 2). $\mathrm{KCl}$ at $0.5 \%$ showed the significantly lowest spore germination i.e. $57.06 \%$ followed by $\mathrm{CaSO}_{4}$ at $0.5 \%$ $(59.50 \%)$ and $\mathrm{K}_{2} \mathrm{SO}_{4}$ at $0.5 \%(62.20 \%)$. All chemicals except borax were found significant over check. The minimum per cent reduction of spore germination over check was recorded in borax at $0.5 \%(3.48 \%)$ and $\mathrm{ZnSO}_{4}$ at $0.5 \%(12.78 \%)$.

All the treatments showed increase of sporulation intensity except $\mathrm{CaSO}_{4}$ at $0.5 \%, \mathrm{~K}_{2} \mathrm{SO}_{4}$ at $0.5 \%$ and $\mathrm{ZnSO}_{4}$ at $0.75 \%$ (Table 3 ). The minimum sporulation was recorded in $\mathrm{CaSO}_{4}$ at $0.5 \%$ in which about $40.18 \%$ reduction was observed as compared to check. The maximum sporulation was observed in treatment $\mathrm{K}_{2} \mathrm{SO}_{4}$ at $1.0 \%\left(93.12 \times 10^{3}\right.$ spores $/ \mathrm{ml}$ ) followed by $\mathrm{Na}_{2} \mathrm{~B}_{4} \mathrm{O}_{7} .10 \mathrm{H}_{2} \mathrm{O}$ at $0.5 \%$ $\left(44.50 \times 10^{3}\right.$ spores $\left./ \mathrm{ml}\right)$. None of the chemicals was found significantly affecting the sporulation intensity in comparison to check except $\mathrm{K}_{2} \mathrm{SO}_{4}$ at $1.0 \%$ and $\mathrm{Na}_{2} \mathrm{~B}_{4} \mathrm{O}_{7} .10 \mathrm{H}_{2} \mathrm{O}$ at $0.5 \%$.

Kaur (2000) observed that $\mathrm{ZnSO}_{4}$ was found to be inhibitory to Albugo candida, Peronospora parasitica and $A$. brassicae. Furuya et al. (1999) also found no germination of Fusarium oxysporum f sp. Phaseoli in soil with higher aluminium contents. It may be chance that heavy ions of metal and non-metal inactivate enzymes required for germination. Vidhyasekaran (1988) reported

Table 1. Effect of different concentrations of some eco-friendly chemicals and mancozeb on the growth of $A$. brassicae on potato sucrose agar (PSA) medium.

\begin{tabular}{lccccc}
\hline \multirow{2}{*}{ Treatment } & $\begin{array}{c}\text { Concentration } \\
(\mathbf{p p m})\end{array}$ & $\mathbf{7 ~ D A I}$ & Inhibition (\%) & 14 DAI & Inhibition (\%) \\
\cline { 2 - 5 } & 100 & 3.53 & 41.16 & 5.30 & 29.89 \\
$\mathrm{CaSO}_{4}$ & 500 & 3.26 & 45.66 & 4.76 & 37.03 \\
& 1000 & 2.96 & 50.66 & 4.43 & 41.40 \\
& 100 & 2.73 & 54.50 & 3.53 & 53.30 \\
$\mathrm{KCl}$ & 500 & 2.53 & 57.83 & 3.23 & 57.27 \\
& 1000 & 2.23 & 62.83 & 3.10 & 58.99 \\
$\mathrm{~K}_{2} \mathrm{SO}_{4}$ & 100 & 3.20 & 46.66 & 3.90 & 48.41 \\
& 500 & 2.56 & 57.33 & 3.43 & 54.62 \\
$\mathrm{ZnSO}_{4}$ & 1000 & 2.20 & 63.33 & 2.70 & 64.28 \\
& 100 & 3.10 & 48.33 & 4.56 & 39.68 \\
& 500 & 2.13 & 64.50 & 3.80 & 49.73 \\
$\mathrm{Na}_{2} \mathrm{~B}_{4} \mathrm{O}_{7} .10 \mathrm{H}_{2} \mathrm{O}$ & 1000 & 1.33 & 77.83 & 2.73 & 63.88 \\
& 100 & 4.16 & 30.66 & 5.40 & 28.57 \\
& 500 & 3.40 & 43.33 & 4.40 & 41.79 \\
$\mathrm{Mancozeb}$ & 1000 & 3.20 & 46.66 & 3.93 & 48.01 \\
$\mathrm{Check}$ & 100 & 1.86 & 69.00 & 4.26 & 43.65 \\
\hline
\end{tabular}

CD- Critical difference; DAI = Days after inoculation. 
Table 2. Effect of some eco-friendly chemicals on spore germination of $A$. brassicae.

\begin{tabular}{lccc}
\hline Treatment & Concentration (\%) & Spore germination after 8 hrs (\%) & Reduction over check (\%) \\
\hline $\mathrm{CaSO}_{4}$ & 0.5 & 59.50 & 20.24 \\
$\mathrm{KCl}$ & 0.5 & 57.06 & 23.51 \\
$\mathrm{~K}_{2} \mathrm{SO}_{4}$ & 0.5 & 62.20 & 16.62 \\
$\mathrm{ZnSO}_{4}$ & 0.5 & 65.06 & 12.78 \\
$\mathrm{Na}_{2} \mathrm{~B}_{4} \mathrm{O}_{7} .10 \mathrm{H}_{2} \mathrm{O}$ & 0.5 & 72.00 & 3.48 \\
\hline $\mathrm{Check}$ & - & 74.60 & - \\
\hline C.D. at $5 \%$ & & 3.56 & \\
\hline
\end{tabular}

CD- Critical difference

Table 3. Effect of some eco-friendly chemicals on intensity of sporulation of A. brassicae on naturally inoculated leaves of mustard cv. Varuna.

\begin{tabular}{lccc}
\hline Treatment & Concentration (\%) & $\begin{array}{c}\text { Spore intensity } \\
\left(\times \mathbf{1 0}^{\mathbf{3}} / \mathbf{m l}\right)\end{array}$ & $\begin{array}{c}\text { Per cent increase (+) or decrease (-) } \\
\text { over check }\end{array}$ \\
\hline & 0.5 & 7.25 & -40.18 \\
$\mathrm{CaSO}_{4}$ & 1.0 & 14.50 & +19.63 \\
& 1.5 & 33.25 & +174.33 \\
& 0.5 & 29.25 & +141.33 \\
$\mathrm{KCl}$ & 1.0 & 17.25 & +42.32 \\
& 1.5 & 24.50 & +102.14 \\
& 0.5 & 8.50 & -29.86 \\
$\mathrm{~K}_{2} \mathrm{SO}_{4}$ & 1.0 & 93.12 & +668.31 \\
& 1.5 & 37.25 & +207.34 \\
& 0.25 & 21.00 & +73.26 \\
$\mathrm{ZnSO}_{4}$ & 0.50 & 36.00 & +197.02 \\
& 0.75 & 11.50 & -5.11 \\
$\mathrm{Na}_{2} \mathrm{~B}_{4} \mathrm{O}_{7} .10 \mathrm{H}_{2} \mathrm{O}$ & 0.25 & 20.00 & +65.01 \\
& 0.50 & 44.50 & +267.16 \\
& 0.75 & 12.75 & +5.19 \\
\hline $\mathrm{Check}$ & - & 12.12 & - \\
\hline $\mathrm{C} . \mathrm{D}$. at $5 \%$ & & 26.53 & \\
\hline
\end{tabular}

CD- Critical difference

that calcium polypectates are resistant to hydrolysis by pectolytic enzymes produced by pathogens. The results from such studies revealed that the differences in spore germination and sporulation intensity may be due to activation of various inducing principles, which might have resulted in various PR proteins, phenolics, phytoalexins and other toxic or stimulatory metabolites (Marschner, 1986; Rouxel et al., 1995).

In vivo evaluation of eco-friendly chemicals against A. brassicae: The percent disease index on leaves was observed at 60, 70, 80 and 90 DAS (Table 4). The leaf disease index was found to be highly significant among the treatments, observation intervals and their interactions. The significantly maximum reduction of leaf disease index was recorded in $\mathrm{CaSO}_{4}$ at $0.5 \%$ (36.96 per cent) followed by $\mathrm{CaSO}_{4}$ at $1.5 \%$ (35.84\%) and $\mathrm{Na}_{2} \mathrm{~B}_{4} \mathrm{O}_{7} .10 \mathrm{H}_{2} \mathrm{O}$ at $0.75 \%(35.63 \%)$ in comparison to check. The leaf disease index was observed maximum in all the treatments of $\mathrm{ZnSO}_{4}$ i.e. at $0.25 \%(35.00 \%)$,
$0.5 \% \quad(32.91 \%)$ and $0.75 \%(31.91 \%)$ respectively. Among the borax, the maximum leaf disease index was found at $0.5 \%(28.50 \%)$ followed by $0.25 \%$ $(27.41 \%)$ and $0.75 \%(24.08 \%)$, respectively.

The percent disease index on pods was observed at 80,90 , 100 and 110 DAS (Table 5). The significantly maximum reduction of disease index on pod was found in $\mathrm{Na}_{2} \mathrm{~B}_{4} \mathrm{O}_{7} .10 \mathrm{H}_{2} \mathrm{O}$ at $0.75 \%$ (37.62\%) followed by $\mathrm{K}_{2} \mathrm{SO}_{4}$ at $1.5 \%(32.82 \%)$ and $\mathrm{KCl}$ at $1.5 \%(32.16 \%)$ over check. The disease index was to be highly significant among the treatments, observation intervals and their interaction. Among $\mathrm{CaSO}_{4}$ treatments, the minimum disease index on pod was observed at $1.0 \%$ (27.91\%) followed by $0.5 \%(31.91 \%)$ and $1.5 \%$ (32.08\%), respectively.

The significantly maximum and minimum numbers of primary branches were observed in $\mathrm{CaSO}_{4}$ at $1.0 \%$ (7.00) and $\mathrm{CaSO}_{4}$ at $1.5 \%$ (5.26), respectively. There was significant effect on number of primary branches due 
Table 4. Effect of some eco-friendly chemicals on disease severity of alternaria blight on leaf at different stages of growth of mustard cv. Varuna.

\begin{tabular}{|c|c|c|c|c|c|c|c|}
\hline \multirow[t]{2}{*}{ Treatment } & \multirow{2}{*}{$\begin{array}{c}\text { Concentration } \\
(\%)\end{array}$} & \multicolumn{4}{|c|}{ Disease index (\%) } & \multirow[t]{2}{*}{ Mean } & \multirow{2}{*}{$\begin{array}{l}\text { Reduction } \\
\text { over check } \\
\text { (\%) }\end{array}$} \\
\hline & & 60 DAS & 70 DAS & 80 DAS & 90 DAS & & \\
\hline \multirow{3}{*}{$\mathrm{CaSO}_{4}$} & 0.5 & $\begin{array}{c}2.00 \\
(8.13)\end{array}$ & $\begin{array}{c}10.33 \\
(18.72)\end{array}$ & $\begin{array}{c}35.66 \\
(36.53)\end{array}$ & $\begin{array}{c}46.33 \\
(42.89)\end{array}$ & $\begin{array}{l}23.58 \\
(26.59)\end{array}$ & 36.96 \\
\hline & 1.0 & $\begin{array}{c}4.00 \\
(11.47)\end{array}$ & $\begin{array}{c}12.66 \\
(20.73)\end{array}$ & $\begin{array}{c}47.33 \\
(43.47)\end{array}$ & $\begin{array}{c}56.66 \\
(48.83)\end{array}$ & $\begin{array}{l}30.16 \\
(31.13)\end{array}$ & 19.37 \\
\hline & 1.5 & $\begin{array}{c}4.33 \\
(11.99)\end{array}$ & $\begin{array}{c}11.33 \\
(19.65)\end{array}$ & $\begin{array}{c}33.33 \\
(35.22)\end{array}$ & $\begin{array}{c}47.00 \\
(43.27)\end{array}$ & $\begin{array}{l}24.00 \\
(27.54)\end{array}$ & 35.84 \\
\hline \multirow{3}{*}{$\mathrm{KCl}$} & 0.5 & $\begin{array}{c}3.33 \\
(10.49)\end{array}$ & $\begin{array}{c}10.66 \\
(18.98)\end{array}$ & $\begin{array}{c}43.66 \\
(41.35)\end{array}$ & $\begin{array}{c}60.00 \\
(50.77)\end{array}$ & $\begin{array}{c}29.41 \\
(30.40)\end{array}$ & 21.38 \\
\hline & 1.0 & $\begin{array}{c}3.66 \\
(11.01)\end{array}$ & $\begin{array}{c}13.00 \\
(21.12)\end{array}$ & $\begin{array}{c}39.33 \\
(38.83)\end{array}$ & $\begin{array}{c}62.66 \\
(52.34)\end{array}$ & $\begin{array}{c}29.66 \\
(30.83)\end{array}$ & 20.71 \\
\hline & 1.5 & $\begin{array}{c}3.00 \\
(9.88)\end{array}$ & $\begin{array}{c}13.33 \\
(21.26)\end{array}$ & $\begin{array}{c}43.33 \\
(41.16)\end{array}$ & $\begin{array}{c}62.00 \\
(51.95)\end{array}$ & $\begin{array}{c}30.41 \\
(31.06)\end{array}$ & 18.71 \\
\hline \multirow{3}{*}{$\mathrm{K}_{2} \mathrm{SO}_{4}$} & 0.5 & $\begin{array}{c}4.33 \\
(11.99)\end{array}$ & $\begin{array}{c}10.33 \\
(18.73)\end{array}$ & $\begin{array}{c}38.33 \\
(38.24)\end{array}$ & $\begin{array}{c}63.66 \\
(52.63)\end{array}$ & $\begin{array}{l}29.16 \\
(30.47)\end{array}$ & 22.05 \\
\hline & 1.0 & $\begin{array}{c}3.66 \\
(10.95)\end{array}$ & $\begin{array}{c}10.66 \\
(19.03)\end{array}$ & $\begin{array}{c}39.00 \\
(38.64)\end{array}$ & $\begin{array}{c}63.00 \\
(52.53)\end{array}$ & $\begin{array}{l}29.08 \\
(30.29)\end{array}$ & 22.26 \\
\hline & 1.5 & $\begin{array}{c}2.66 \\
(9.35)\end{array}$ & $\begin{array}{c}12.66 \\
(20.83)\end{array}$ & $\begin{array}{c}45.00 \\
(42.12)\end{array}$ & $\begin{array}{c}65.66 \\
(54.13)\end{array}$ & $\begin{array}{c}31.50 \\
(31.61)\end{array}$ & 15.79 \\
\hline \multirow{3}{*}{$\mathrm{ZnSO}_{4}$} & 0.25 & $\begin{array}{c}4.33 \\
(11.99)\end{array}$ & $\begin{array}{c}13.66 \\
(21.67)\end{array}$ & $\begin{array}{c}54.33 \\
(47.48)\end{array}$ & $\begin{array}{c}67.66 \\
(55.34)\end{array}$ & $\begin{array}{c}35.00 \\
(34.12)\end{array}$ & 6.44 \\
\hline & 0.50 & $\begin{array}{l}2.33 \\
(8.74)\end{array}$ & $\begin{array}{c}12.00 \\
(20.22)\end{array}$ & $\begin{array}{c}52.33 \\
(46.33)\end{array}$ & $\begin{array}{c}65.00 \\
(53.73)\end{array}$ & $\begin{array}{c}32.91 \\
(32.26)\end{array}$ & 12.02 \\
\hline & 0.75 & $\begin{array}{c}3.33 \\
(10.49)\end{array}$ & $\begin{array}{c}14.00 \\
(21.12)\end{array}$ & $\begin{array}{c}48.66 \\
(44.23)\end{array}$ & $\begin{array}{c}62.66 \\
(52.33)\end{array}$ & $\begin{array}{c}31.91 \\
(32.04)\end{array}$ & 14.70 \\
\hline \multirow{3}{*}{$\mathrm{Na}_{2} \mathrm{~B}_{4} \mathrm{O}_{7} \cdot 10 \mathrm{H}_{2} \mathrm{O}$} & 0.25 & $\begin{array}{c}2.33 \\
(11.01)\end{array}$ & $\begin{array}{c}10.00 \\
(18.42)\end{array}$ & $\begin{array}{c}44.33 \\
(41.74)\end{array}$ & $\begin{array}{c}53.00 \\
(46.72)\end{array}$ & $\begin{array}{c}27.41 \\
(28.90)\end{array}$ & 26.73 \\
\hline & 0.50 & $\begin{array}{c}3.66 \\
(11.01)\end{array}$ & $\begin{array}{c}8.66 \\
(17.07)\end{array}$ & $\begin{array}{c}39.00 \\
(38.61)\end{array}$ & $\begin{array}{c}62.66 \\
(52.33)\end{array}$ & $\begin{array}{l}28.50 \\
(29.76)\end{array}$ & 23.81 \\
\hline & 0.75 & $\begin{array}{c}4.00 \\
(11.47) \\
\end{array}$ & $\begin{array}{c}12.66 \\
(20.80) \\
\end{array}$ & $\begin{array}{r}32.00 \\
(34.44) \\
\end{array}$ & $\begin{array}{c}47.66 \\
(43.66) \\
\end{array}$ & $\begin{array}{c}24.08 \\
(27.59) \\
\end{array}$ & 35.63 \\
\hline Check & - & $\begin{array}{c}6.33 \\
(14.56)\end{array}$ & $\begin{array}{c}17.00 \\
(24.30)\end{array}$ & $\begin{array}{c}55.33 \\
(48.06)\end{array}$ & $\begin{array}{c}71.00 \\
(57.42)\end{array}$ & $\begin{array}{c}37.41 \\
(36.09)\end{array}$ & - \\
\hline Mean & & $\begin{array}{c}3.58 \\
(10.77) \\
\end{array}$ & $\begin{array}{c}12.00 \\
(20.17)\end{array}$ & $\begin{array}{c}43.18 \\
(41.04)\end{array}$ & $\begin{array}{c}59.79 \\
(50.70) \\
\end{array}$ & & \\
\hline $\begin{array}{l}\text { C.D. at 5\% } \\
\text { Treatment } \\
\text { Interval } \\
\text { Interaction }\end{array}$ & & & & & & $\begin{array}{l}1.69 \\
0.84 \\
3.38 \\
\end{array}$ & \\
\hline
\end{tabular}

CD- Critical difference; Figure in parentheses indicates angular transformed values.

to spray of non-conventional chemicals (Table 6). None of the non-conventional chemicals were found significantly affecting the number of secondary branches and number of seeds/plant. There was significant effect on number of siliqua/plant by spraying of nonconventional chemicals (Table 6). The maximum number of siliqua/ plant was found in treatment $\mathrm{KCl}$ at $1.0 \%$ (748.39) followed by $\mathrm{K}_{2} \mathrm{SO}_{4}$ at $1.5 \%$ (744.24) and $\mathrm{CaSO}_{4}$ at $0.5 \%(712.51)$. The total seed yield/ha increased in all the treatments over check except $\mathrm{K}_{2} \mathrm{SO}_{4}$ at $0.5 \%$ $(1339.06 \mathrm{~kg} / \mathrm{ha})$. Among the treatments, maximum seed yield/ha was recorded in case of $\mathrm{CaSO}_{4}$ at $1.0 \%$ $\left(1917.30 \mathrm{~kg} / \mathrm{ha}\right.$ ) followed by $\mathrm{Na}_{2} \mathrm{~B}_{4} \mathrm{O}_{7} .10 \mathrm{H}_{2} \mathrm{O}$ at $0.75 \%$ $(1642.26 \mathrm{~kg} / \mathrm{ha})$ and $\mathrm{CaSO}_{4}$ at $1.5 \%(1596.36 \mathrm{~kg} / \mathrm{ha})$. There was no significant effect of any nonconventional chemicals on oil content. However, the maximum oil content was observed in case of $\mathrm{Na}_{2} \mathrm{~B}_{4} \mathrm{O}_{7} \cdot 10 \mathrm{H}_{2} \mathrm{O}$ at $0.75 \%$ (39.75 per cent) in comparison to check (37.20 
Table 5. Effect of some eco-friendly chemicals on disease severity of Alternaria blight on pod at different stages of growth of mustard cv. Varuna.

\begin{tabular}{|c|c|c|c|c|c|c|c|}
\hline \multirow[t]{2}{*}{ Treatment } & \multirow[t]{2}{*}{$\begin{array}{c}\text { Concentration } \\
(\%)\end{array}$} & \multicolumn{4}{|c|}{$\begin{array}{c}\text { Disease index } \\
(\%)\end{array}$} & \multirow[t]{2}{*}{ Mean } & \multirow{2}{*}{$\begin{array}{l}\text { Reduction } \\
\text { over check } \\
\text { (\%) }\end{array}$} \\
\hline & & 80 DAS & 90 DAS & 100 DAS & 110 DAS & & \\
\hline \multirow{3}{*}{$\mathrm{CaSO}_{4}$} & 0.5 & $\begin{array}{c}7.00 \\
(15.31)\end{array}$ & $\begin{array}{l}21.00 \\
(27.25)\end{array}$ & $\begin{array}{l}45.66 \\
(42.48)\end{array}$ & $\begin{array}{l}54.00 \\
(47.29)\end{array}$ & $\begin{array}{c}31.91 \\
(33.08)\end{array}$ & 16.74 \\
\hline & 1.0 & $\begin{array}{c}4.66 \\
(12.35)\end{array}$ & $\begin{array}{c}17.33 \\
(24.59)\end{array}$ & $\begin{array}{l}39.66 \\
(39.03)\end{array}$ & $\begin{array}{c}50.00 \\
(44.99)\end{array}$ & $\begin{array}{c}27.91 \\
(30.24)\end{array}$ & 27.18 \\
\hline & 1.5 & $\begin{array}{c}5.33 \\
(13.34)\end{array}$ & $\begin{array}{c}22.33 \\
(28.19)\end{array}$ & $\begin{array}{l}43.66 \\
(41.35)\end{array}$ & $\begin{array}{c}57.00 \\
(49.02)\end{array}$ & $\begin{array}{c}32.08 \\
(32.98)\end{array}$ & 16.30 \\
\hline \multirow{3}{*}{$\mathrm{KCl}$} & 0.5 & $\begin{array}{c}4.00 \\
(11.47)\end{array}$ & $\begin{array}{c}17.66 \\
(24.84)\end{array}$ & $\begin{array}{l}39.66 \\
(39.02)\end{array}$ & $\begin{array}{c}51.66 \\
(45.95)\end{array}$ & $\begin{array}{c}28.25 \\
(30.32)\end{array}$ & 26.29 \\
\hline & 1.0 & $\begin{array}{c}7.33 \\
(15.67)\end{array}$ & $\begin{array}{c}21.66 \\
(27.73)\end{array}$ & $\begin{array}{l}43.00 \\
(40.97)\end{array}$ & $\begin{array}{c}56.33 \\
(48.64)\end{array}$ & $\begin{array}{c}32.08 \\
(33.25)\end{array}$ & 16.30 \\
\hline & 1.5 & $\begin{array}{c}5.66 \\
(13.75)\end{array}$ & $\begin{array}{l}19.66 \\
(26.30)\end{array}$ & $\begin{array}{l}31.00 \\
(33.81)\end{array}$ & $\begin{array}{c}47.66 \\
(43.66)\end{array}$ & $\begin{array}{c}26.00 \\
(29.38)\end{array}$ & 32.16 \\
\hline \multirow{3}{*}{$\mathrm{K}_{2} \mathrm{SO}_{4}$} & 0.5 & $\begin{array}{c}9.00 \\
(17.38)\end{array}$ & $\begin{array}{c}25.66 \\
(30.38)\end{array}$ & $\begin{array}{l}32.66 \\
(34.85)\end{array}$ & $\begin{array}{c}46.33 \\
(42.89)\end{array}$ & $\begin{array}{c}28.41 \\
(31.38)\end{array}$ & 25.88 \\
\hline & 1.0 & $\begin{array}{c}7.66 \\
(16.04)\end{array}$ & $\begin{array}{c}22.66 \\
(28.42)\end{array}$ & $\begin{array}{l}39.66 \\
(39.03)\end{array}$ & $\begin{array}{c}57.00 \\
(49.02)\end{array}$ & $\begin{array}{c}31.75 \\
(33.13)\end{array}$ & 17.16 \\
\hline & 1.5 & $\begin{array}{c}7.00 \\
(15.31)\end{array}$ & $\begin{array}{c}18.66 \\
(25.57)\end{array}$ & $\begin{array}{l}30.66 \\
(33.62)\end{array}$ & $\begin{array}{c}46.66 \\
(43.08)\end{array}$ & $\begin{array}{c}25.75 \\
(29.40)\end{array}$ & 32.82 \\
\hline \multirow{3}{*}{$\mathrm{ZnSO}_{4}$} & 0.25 & $\begin{array}{c}7.33 \\
(15.65)\end{array}$ & $\begin{array}{c}19.00 \\
(25.83)\end{array}$ & $\begin{array}{c}44.66 \\
(41.93)\end{array}$ & $\begin{array}{c}58.00 \\
(49.60)\end{array}$ & $\begin{array}{c}32.25 \\
(33.25)\end{array}$ & 15.86 \\
\hline & 0.50 & $\begin{array}{c}7.00 \\
(15.24)\end{array}$ & $\begin{array}{c}16.66 \\
(24.06)\end{array}$ & $\begin{array}{l}35.00 \\
(36.26)\end{array}$ & $\begin{array}{c}55.33 \\
(48.06)\end{array}$ & $\begin{array}{c}28.50 \\
(30.90)\end{array}$ & 25.64 \\
\hline & 0.75 & $\begin{array}{c}5.66 \\
(13.68)\end{array}$ & $\begin{array}{c}21.66 \\
(27.73)\end{array}$ & $\begin{array}{l}30.00 \\
(33.18)\end{array}$ & $\begin{array}{c}54.66 \\
(47.67)\end{array}$ & $\begin{array}{c}28.00 \\
(30.57)\end{array}$ & 26.95 \\
\hline \multirow{3}{*}{$\mathrm{Na}_{2} \mathrm{~B}_{4} \mathrm{O}_{7} \cdot 10 \mathrm{H}_{2} \mathrm{O}$} & 0.25 & $\begin{array}{c}9.00 \\
(17.44)\end{array}$ & $\begin{array}{c}17.66 \\
(24.88)\end{array}$ & $\begin{array}{l}29.66 \\
(32.99)\end{array}$ & $\begin{array}{c}49.33 \\
(44.61)\end{array}$ & $\begin{array}{c}26.71 \\
(29.97)\end{array}$ & 30.31 \\
\hline & 0.50 & $\begin{array}{c}7.66 \\
(15.98)\end{array}$ & $\begin{array}{l}22.00 \\
(27.96)\end{array}$ & $\begin{array}{c}34.33 \\
(35.84)\end{array}$ & $\begin{array}{c}52.33 \\
(46.33)\end{array}$ & $\begin{array}{c}29.08 \\
(31.53)\end{array}$ & 24.13 \\
\hline & 0.75 & $\begin{array}{c}5.33 \\
(13.29)\end{array}$ & $\begin{array}{c}15.33 \\
(22.89)\end{array}$ & $\begin{array}{c}30.00 \\
(33.19)\end{array}$ & $\begin{array}{c}45.00 \\
(42.12)\end{array}$ & $\begin{array}{c}23.91 \\
(27.87)\end{array}$ & 37.62 \\
\hline Check & - & $\begin{array}{c}10.66 \\
(19.03) \\
\end{array}$ & $\begin{array}{c}28.00 \\
(31.91) \\
\end{array}$ & $\begin{array}{c}48.00 \\
(43.85) \\
\end{array}$ & $\begin{array}{c}66.66 \\
(54.74) \\
\end{array}$ & $\begin{array}{c}38.33 \\
(37.38) \\
\end{array}$ & - \\
\hline Mean & & $\begin{array}{c}6.89 \\
(15.06) \\
\end{array}$ & $\begin{array}{c}20.43 \\
(26.78) \\
\end{array}$ & $\begin{array}{c}37.33 \\
(37.59) \\
\end{array}$ & $\begin{array}{c}53.00 \\
(46.73) \\
\end{array}$ & & \\
\hline $\begin{array}{l}\text { C.D. at 5\% } \\
\text { Treatment } \\
\text { Interval } \\
\text { Interaction }\end{array}$ & & & & & & $\begin{array}{l}1.99 \\
0.99 \\
3.99 \\
\end{array}$ & \\
\hline
\end{tabular}

CD- Critical difference; Figure in parentheses indicates angular transformed values.

per cent) (Table 6).

Graham (1983) reported that macro and micronutrients play an important role in protection by affecting plant susceptibility to pathogens. They may also affect the predisposition of plants to viral diseases, which have been reported to increase or decrease the resistance of asparagus bean to tobacco necrosis virus (Pennazio and Roggero, 1988). However, the induction of resistance to foliar pathogens by a foliar spray of different concentrations of micronutrient solutions, has not been extensively investigated. The most important feature of these elements in this regard, is their variable valency, which allows them to be involved in oxidation changes and they, therefore, serve to function as co-factor of metalo -protein enzymes such as peroxidase and b-1,3 glucanase (Reuveni et al., 1997).

The present investigation supported the findings of Meena et al. (2011) that showed that calcium sulphate, 
Table 6. Effect of some eco-friendly chemicals on growth components and yield of mustard cv. Varuna.

\begin{tabular}{|c|c|c|c|c|c|c|c|}
\hline \multirow[b]{2}{*}{ Treatment } & \multirow[b]{2}{*}{$\begin{array}{c}\text { Concentration } \\
(\%)\end{array}$} & \multicolumn{6}{|c|}{ Plant growth and yield components } \\
\hline & & $\begin{array}{c}\text { No. of } \\
\text { primary } \\
\text { branches }\end{array}$ & $\begin{array}{c}\text { No. of } \\
\text { secondary } \\
\text { branches } \\
\end{array}$ & $\begin{array}{c}\text { No. of } \\
\text { siliqua/ } \\
\text { plant }\end{array}$ & $\begin{array}{l}\text { No. of } \\
\text { seeds/ } \\
\text { plant } \\
\end{array}$ & $\begin{array}{c}\text { Total seed } \\
\text { yield/ha } \\
(\mathrm{kg}) \\
\end{array}$ & $\begin{array}{c}\text { Oil } \\
\text { content } \\
(\%)\end{array}$ \\
\hline \multirow{3}{*}{$\mathrm{CaSO}_{4}$} & 0.5 & 5.73 & 9.86 & 712.51 & 14.06 & 1560.40 & 39.25 \\
\hline & 1.0 & 7.00 & 13.00 & 628.53 & 13.40 & 1917.30 & 38.97 \\
\hline & 1.5 & 5.26 & 9.93 & 451.95 & 11.26 & 1596.36 & 39.02 \\
\hline \multirow{3}{*}{$\mathrm{KCl}$} & 0.5 & 5.46 & 11.46 & 442.22 & 14.66 & 1491.23 & 39.21 \\
\hline & 1.0 & 5.73 & 9.73 & 748.39 & 14.06 & 1510.60 & 39.24 \\
\hline & 1.5 & 5.40 & 10.20 & 466.10 & 13.86 & 1505.06 & 38.97 \\
\hline \multirow{3}{*}{$\mathrm{K}_{2} \mathrm{SO}_{4}$} & 0.5 & 6.00 & 9.40 & 352.49 & 13.93 & 1339.06 & 39.84 \\
\hline & 1.0 & 6.40 & 11.53 & 710.99 & 13.80 & 1538.26 & 39.38 \\
\hline & 1.5 & 5.40 & 9.99 & 744.24 & 13.80 & 1449.73 & 38.90 \\
\hline \multirow{3}{*}{$\mathrm{ZnSO}_{4}$} & 0.25 & 6.00 & 9.40 & 454.06 & 13.13 & 1397.16 & 39.59 \\
\hline & 0.50 & 5.86 & 11.60 & 402.49 & 12.66 & 1330.76 & 38.81 \\
\hline & 0.75 & 6.26 & 11.20 & 658.00 & 13.00 & 1510.60 & 38.72 \\
\hline \multirow{3}{*}{$\begin{array}{l}\mathrm{Na}_{2} \mathrm{~B}_{4} \mathrm{O}_{7} \\
10 \mathrm{H}_{2} \mathrm{O}\end{array}$} & 0.25 & 6.06 & 11.60 & 568.11 & 13.80 & 1427.60 & 39.28 \\
\hline & 0.50 & 5.73 & 11.20 & 488.02 & 12.93 & 1388.86 & 38.85 \\
\hline & 0.75 & 5.86 & 10.20 & 317.99 & 13.60 & 1642.26 & 39.75 \\
\hline Check & - & 6.40 & 7.86 & 592.37 & 13.33 & 1355.66 & 37.20 \\
\hline C.D. at 5\% & & 0.54 & NS & 97.64 & NS & 213.10 & NS \\
\hline
\end{tabular}

CD- Critical difference; NS-Not significant

borax and zinc sulphate were found effective against alternaria blight of Indian mustard. Role of calcium in improving tolerance in plants to diseases have been indicated earlier (Reddy, 2001; Agrios, 2005). The foliar application of $\mathrm{CaSO}_{4}$ at $0.5 \%$ concentration induced resistance significantly against alternaria blight of mustard (Kumar et al., 2014). Tewari (1991) examined the black spot lesions on rapeseed leaves by scanning electron microscopy in conjugation with energy-dispersive X-ray microanalysis and revealed that the sequestration of calcium by $A$. brassicae. Therefore, there are possibilities of enhancing resistance to $A$. brassicae in rapeseed by soil or foliar spray of calcium compounds. In the present investigation, $\mathrm{CaSO}_{4}$ showed remarkable effect on leaf infection but not as much on pod infection. The possible reason for this could be the effect of particular nutrient at different stages of host-pathogen interaction would be likely to be different.

Sharma and Kolte (1994) concluded that under natural conditions, based on the number and size of spot, per cent leaf and pod infection, average disease index on leaf and pod, K-fertilized plants reduced severity of Alternaria blight over N, P and NP fertilized toria plant. Singh (1996) concluded that under field conditions application of $\mathrm{KMnO}_{4}$ and $\mathrm{KAl}\left(\mathrm{SO}_{4}\right)_{2}$ showed reduction in disease index of Alternaria blight of rapeseed. Vishwanath (1987) reported that foliar spray of boric acid (0.53\%) gave 20-64 per cent disease control of Alternaria blight.

Soil applied sulphur was found to increase resistance against a variety of fungal pathogens on different crops (Klikocka et al., 2005). Agrawal (1999) reported that $\mathrm{ZnSO}_{4}$ sprayed plants showed maximum grain yield and 1000-grain weight in maize as compared to check. Foliar sprays of Zinc Sulpate at $10^{-3} \mathrm{mmol}$ to chickpea (Cicer arietinum) and subsequently challenged against Sclerotinia sclerotiorum, causing stem rot in chickpea gave the best result and only $13.6 \%$ mortality was recorded after 28 days as compared to $100 \%$ in the control (Sarma et al., 2007).

In the present study the treatment $\mathrm{CaSO}_{4}$ at $1.0 \%$ significantly increased the number of primary branches, number of secondary branches, seed yield/plant and total yield/ha (Table 6). Calcium has critical roles in cell division, cell development, carbohydrate movement, neutralization of cell acids, cell wall deposition and formation of pectate salts in the middle lamella (Huber and Arny, 1985). 


\section{Conclusion}

For plant disease management, increasing public concern about environment and health is proving to be major hindrance in the use of chemical fungicides. Implications of harmful chemicals strongly necessitates the search the new eco-friendly methods for plant disease management. This study showed that potassium sulphate showed maximum in vitro effect on Alternaria brassicae and calcium sulphate and borax are providing maximum reduction of disease and increase in seed yield/ha in field condition. Therefore, the eco-friendly technologies reported here could help the farmers of India for management of alternaria blight disease and could increase the production and productivity of the Indian mustard crop.

\section{REFERENCES}

Agrios, G.N. (2005). Plant pathology, $5^{\text {th }}$ Edn., Elsevier Academic Publishers, California, USA, pp 922.

Agrawal, N. (1999). Role of some nutrients, organic acids, growth regulators and agro-meteorological factors on the severity of brown strip downy mildew of maize. Thesis, Ph.D. (Plant Pathology), G.B. Pant Univ. of Agric. \& Tech., Pantnagar, pp 199.

Chattopadhyay, C., Agrawal, R., Kumar, A., Bhar, L.M., Meena, P.D., Meena, R.L., Khan, S.A., Chattopadhyay, A.K., Awasthi, R.P., Singh, S.N., Chakravarthy, N.V.K., Kumar, A., Singh, R. B., Bhunia, C. K. (2005). Epidemiology and forecasting of Alternaria blight of oilseed Brassica in India e a case study. Z. Pflanzenkr. Pflanzenschutz. J. Plant Dis. Prot., 112: 351-365.

Conn, K.L., Tewari, J.P., Awasthi, R.P. (1990). A disease assessment key for Alternaria blackspot in rapeseed and mustard. Can. Plant Dis. Surv., 70: 19-22.

Furuya, H.; Takahashi, T. and Matsumoto, T. (1999). Suppression of Fusarium solani f. sp. Phaseoli on bean by aluminium in acid soils. Phytopath., 89: 47-52.

Gattani, M.L. (1954). Agar plate spore germination method for testing fungicides. Phytopath. $44: 113-115$.

Graham, R.D. (1983). Effect of nutrient stress on susceptibility of plants to disease with particular reference to the trace elements. Adv. Bot. Res., 10: 221-276.

GoI. (2011). Agricultural Statistics at a Glance-2011, Directorate of Economics and Statistics, Ministry of Agriculture, Government of India.

Huber, D.M. and Arny, D.C. (1985). Interactions of potassium with plant disease, In: Munson, R.D. ed. Potassium in agriculture. Madison, Wisconsin, American Society of Agronomy, 667-688.

Kaur, A. (2000). Characterization on biotically and abiotically induced response of mustard against Albugo candida, Peronospora parasitica and Alternaria brassicae. Thesis, Ph.D. (Plant Pathology), G.B. Pant Univ. of Agric. \& Tech., Pantnagar, pp 148.

Kaushik, C.D., Kaushik, J.C. and Saharan, G.S. (1984). Field evaluation of fungicides for the control of Alternaria leaf blight of Brassica juncea. Indian J. Mycol. Pl. Pathol., 9-18.

Khan, M.M., Khan, R.U. and Mohiddin, F.A. (2007). Studies on the cost-effective management of alternaria blight of rapeseed-mustard (Brassica spp.) Phytopathologia Mediterranea, 46: 201-206.

Klikocka, H., Haneklaus, S., Bloem, E. and Schnug, E.
(2005). Influence of sulfur fertilization on infection of potato tubers with Rhizoctonia solani and Streptomyces scabies. Journal of Plant Nutrition. 28: 819-833.

Kolte, S. J., Awasthi, R.P. and Vishwanath (1987). Assessment of yield losses due to Alternaria blight in rapeseed and mustard. Indian Phytopath., 40: 209-211.

Kumar, A., Kumar, S., Kumar, R., Chand, G. and Kolte, S.J. (2014). Fungicidal effect of some non-conventional chemicals for management of alternaria blight disease of mustard. J. Appl. \& Nat. Sci., 6: 913-919.

Marschner, H. (1986). Relationship between mineral nutrition and plant diseases and pests. In : Mineral Nutrition of Higher Plants. Academic Press, New York, pp. 369-390.

McKinney, H.H. (1923). Influence of soil temperature and moisture on infection of wheat seedlings by Helminthosporium sativum. Journal of Agricultural Research, 26: 195-217.

Meena, P.D., Meena, R.L., Chattopadhyay, C. and Kumar, A. (2004). Identification of critical stage for disease development and biocontrol of Alternaria blight of Indian mustard (Brassica juncea). J. Phytopathol., 152: 204-209.

Meena, P.D., Rani, A., Meena, R., Sharma, P., Gupta, R. and Chowdappa, P. (2012). Aggressiveness, diversity and distribution of Alternaria brassicae isolates infecting oilseed Brassica in India. African Journal of Microbiology Research, 6: 5249-5258.

Meena, P.D., Chattopadhyay, C., Kumar, A., Awasthi, R.P., Singh, R., Kaur, S., Thomas, L., Goyal, P. and Chand, P. (2011). Comparative study on the effect of chemicals on alternaria blight in Indian mustard - A multi-location study in India. Journal of Environmental Biology, 32: 375-379.

Meena, P.D., Gour, R.B., Gupta, J.C., Singh, H.K., Awasthi, R.P., Netam, R.S., Godika, S., Sandhu, P.S., Prasad, R., Rathi, A.S., Rai, D., Thomas, L., Patel, G.A. and Chattopadhyay, C. (2013). Non-chemical agents provide tenable, eco-friendly alternatives for the management of the major diseases devastating Indian mustard (Brassica juncea) in India. Crop Protection, 53: 169-174.

Nene, Y.L. and Thapliyal, P.N. (1993). Fungicides in Plant Disease Control. Oxford and IBH Publishing Co., New Delhi, New Delhi, India pp 579.

Pennazio, S. and Roggero, P. (1988). Effect of trace elements on the natural resistance of asparagus bean to tobacco necrosis virus and ethylene production. Advance Hort. Sci., 23-26.

Prasad, R. and Lallu (2006). Management of Alternaria blight of mustard with combination of chemicals and botanicals. Ann. Pl. Protec. Sci., 14: 400-403.

Ram, R.S. and Chauhan, V.B. (1998). Assessment of yield losses due to Alternaria leaf spot in various cultivars of mustard and rapeseed. J. Mycopathol. Res., 36: 109-111.

Reuveni, M., Agapov, V. and Reuveni, R. (1997). A foliar spray of micronutrient solutions induces local and systemic protection against powdery mildew (Sphaerotheca fuliginia) in cucumber. European J. Plant Pathol,. 103: 581-588.

Rouxel, T., Kollmann, A. and Balesdent, M. H. (1995). Phytoalexins from the crucifers. In: Daniel, M. and Purkayastha, R.P. eds. Handbook of Phytoalexin Metabolism and Action. Marcel Decker, New York, pp. 229-261.

Sarma, B.K., Basha, S.A., Singh, D.P. and Singh, U.P. (2007). Use of non-conventional chemicals as an alternative approach to protect chickpea (Cicer arietinum) from Sclerotinia stem rot. Crop Protection, 26: 1042-1048.

Sharma, S. R. and Kolte, S. J. (1994). Effect of soil-applied 
NPK fertilizers on severity of black spot disease (Alternaria brassicae) and yield of oilseed rape. Plant and Soil, 167: 313-320.

Shrestha, S.K., Munk, L. and Mathur, S.B. (2005). Role of weather on Alternaria Leaf Blight Disease and its effect on Yield and Yield Components of Mustard. Nepal Agric. Res. J., 6: 62-72.

Singh, M.P. (1996). Induced host resistance against Alternaria blight, white rust and downy mildew of rapeseed: Role of micronutrients, growth regulators and organic acids. Thesis, Ph.D. (Plant Pathology), G.B. Pant Univ. of Agric. \& Tech., Pantnagar, pp 262.

Sultana, N.A., Khan, M.A.H., Islam, M.N. and Nahar, K. (2009). Evaluation of appropriate time for the application of rovral against alternaria blight incidence and yield of mustard. International Journal of
Sustainable Agriculture, 1: 20-23.

Tewari, J.P. (1991). Structural and biochemical bases of black spot disease of crucifers. Adv. Struct. Biol., 1: 325-349.

Vidhyasekaran, P. (1988). Physiology of Disease Resistance in Plants. Vol. II. Boca Raton, Florida, CRC Press Inc., pp 128.

Verma, P.R. and Saharan, G.S. (1994). Monograph on Alternaria diseases of crucifers. Saskatoon Research Station, Technical Bulletin1994-6E, Agric Agri-Food Canada, Saskatoon, Canada pp 162.

Vishwanath (1987). Studies on some fungicides and micronutrients in the control of three important disease of Toria (Brassica campestris var. toria). Thesis, M.Sc. Ag. (Plant Pathology), G.B. Pant Univ. of Agric. \& Tech., Pantnagar, pp 235. 